\title{
First-principles study of transition-metal nitrides as diffusion barriers against Al
}

\author{
Zhi-Gang Mei*, Abdellatif M. Yacout, Yeon Soo Kim, Gerard Hofman, Marius Stan \\ Argonne National Laboratory, 9700 South Cass Avenue, Argonne, IL 60439 - USA
}

\begin{abstract}
Using density-functional theory based first-principles calculations we provided a comparative study of the diffusion barrier properties of TiN, ZrN, and HfN against Al for U-Mo dispersion fuel applications. We firstly examined the thermodynamic stability of these transition-metal nitrides with $\mathrm{Al}$. The calculated heats of reaction show that both $\mathrm{TiN}$ and $\mathrm{ZrN}$ are thermodynamically unstable diffusion barrier materials, which might be decomposed by $\mathrm{Al}$ at relatively high temperatures. As a comparison, $\mathrm{HfN}$ is a stable diffusion barrier material for Al. To evaluate the kinetic stability of these nitride systems against Al diffusion, we investigated the diffusion mechanisms of $\mathrm{Al}$ in $\mathrm{TiN}, \mathrm{ZrN}$ and $\mathrm{HfN}$ using atomic scale simulations. The effect of non-stoichiometry on the defect formation and Al migration was systematically studied.
\end{abstract}

* Corresponding author. Tel.: +1-630-252-2318.

E-mail address: zmei@anl.gov (Z.G. Mei) 


\section{Introduction}

Uranium-Molybdenum alloy is one of the most promising fuels for future high performance research reactors $[1,2]$. Low-enriched uranium alloys with 6-10 wt\% Mo content are under investigation to replace the high-enriched uranium fuel used by the current research reactors as part of the U.S. Department of Energy, National Nuclear Safety Administration (DOE-NNSA) materials minimization effort. When used in dispersion with aluminum, the growth of inter-diffusion layers (ILs) between the dispersed U-Mo fuel particles and the surrounding Al matrix strongly limits the fuel's performance [3]. The irradiation induced IL not only decreases the thermal conductivity of fuel, but causes the anomalous fuel swelling at high burnups. A major task of U-Mo fuel development is to suppress the formation of IL. One of those proposed approaches is to modify the composition of the Al matrix by adding $\mathrm{Si}$ [4]. It was found that a silicon rich layer formed at the interface between U-Mo and Al prevents the conventional U-Mo/Al inter-diffusion during irradiation. However, excessive fuel swelling can be still observed in aggressive irradiation conditions. Meanwhile, coating U-Mo fuel particles with diffusion barriers was believed to be more efficient in reducing the formation of IL. Recently $\mathrm{ZrN}$ has been tested as a diffusion barrier material for $\mathrm{UMo} / \mathrm{Al}$ dispersion fuel [5, 6]. Post-irradiation-examination (PIE) experiments show that the formation of ILs was significantly reduced and fuel swelling was also reduced due to fewer gas bubbles formed at the UMo-Al interface [7]. However, experiments also reveal that $\mathrm{ZrN}$ coating layers show microcracks during fuel plate fabrication [5] due to its brittle nature. These microcracks in the coating layer can provide fast diffusion channels for $\mathrm{Al}$ migration and therefore fails to protect the fuel particle from direct contact with $\mathrm{Al}$ matrix.

According to Nicolet et al. [8], a good diffusion barrier (DB) should satisfy several criteria: (1) DB layer should be thermodynamically stable against contacting materials; (2) DB layer should constitute a kinetic barrier to the traffic of contacting materials across it; (3) DB should adhere well to substrate and be resistant to thermal and mechanical stresses. For 
nuclear applications, the DB materials should also be stable under irradiation. In practice, these criteria cannot be satisfied simultaneously. Transitional-metal nitrides and their alloys are widely used as coating materials due to their exceptional physical properties, such as excellent thermal stability, hardness and corrosion resistance. A property that is particularly important for microelectronics is the efficiency of these systems to block the migration of $\mathrm{Cu}$ or $\mathrm{Al}$ atoms during metallization of devices $[9,10]$. The diffusion of $\mathrm{Cu}$ in transition-metal nitrides, including $\mathrm{TiN}$ and $\mathrm{ZrN}$, have been studied by Tsetseris et al. using first-principles calculations [11-15]. Recently Pukari et al. also studied the diffusion of inert gas, including $\mathrm{He}, \mathrm{Kr}$ and $\mathrm{Xe}$, in $\mathrm{ZrN}$ from first-principles [16]. However, there are very few experimental studies of $\mathrm{ZrN}$ and $\mathrm{HfN}$ as diffusion barriers against Al. To the authors' best knowledge, there is no theoretical research investigating the diffusion behavior of $\mathrm{Al}$ in $\mathrm{ZrN}$ and $\mathrm{HfN}$.

The purpose of this work is to investigate the thermodynamic and kinetic stability of three transition-metal nitrides (TMNs), i.e., TiN, $\mathrm{ZrN}$ and $\mathrm{HfN}$, as diffusion barrier against $\mathrm{Al}$ using first-principles calculations. This information is expected to be useful to identify new diffusion barrier systems for $\mathrm{U}-\mathrm{Mo} / \mathrm{Al}$ dispersion fuels in the future. The rest of the paper is organized as following. A brief description of the computational methods will be provided in section 2 . The thermodynamic and kinetic stability of the three transition-metal nitrides as diffusion barriers against $\mathrm{Al}$ will be discussed in section 3. In section 4 we will give a summary of this work.

\section{Computational methodology}

The density functional theory (DFT) based first-principles calculations were performed by the projector augmented wave method as implemented in VASP $[17,18]$ within generalized gradient approximation (GGA) parameterized by Perdew, Burke, and Ernzerhof [19]. During the total energy calculations, a plane-wave energy cutoff of $400 \mathrm{eV}$ was employed. The semi-core $s$ and $p$ states were treated as valence electrons for $\mathrm{Ti}$ and $\mathrm{Zr}$, while 
only semi-core $p$ states were included for Hf. In all cases the total energies were converged to at least $10^{-6} \mathrm{eV} /$ atom. TiN, $\mathrm{ZrN}$ and $\mathrm{HfN}$ all adopt the rock salt structure at ambient conditions. The fully relaxed lattice constants are $4.248 \AA$, $4.596 \AA$, and $4.534 \AA$ for TiN, ZrN, and HfN, respectively. The predicted lattice constants agree well will with experimental values of 4.237 , 4.585, and 4.524 ̊ for TiN [20], ZrN [21], and HfN [22], respectively. The obtained lattice parameters were then used to generate $3 \times 3 \times 3$ supercells with $108 \mathrm{TM}$ and $108 \mathrm{~N}$ atoms to study defect formation and Al diffusion in TMNs. The atomic position and cell volume were fully relaxed for all the structures. A Monkhorst-Pack mesh of 3x3x3 k-points in the Brillouin zone is sufficient to satisfy the convergence criterion of the defect formation energy and migration energy, less than $0.02 \mathrm{eV}$.

The formation energy $E_{f}$ of a single defect is calculated with the formula

$$
E_{f}[\mathrm{X}]=E_{\mathrm{tot}}[\mathrm{X}]-E_{\mathrm{tot}}[\mathrm{bulk}]-\sum_{i} n_{i} u_{i}
$$

where $E_{\mathrm{tot}}[\mathrm{X}]$ is the total energy of a supercell containing the defect $\mathrm{X}$, and $E_{\mathrm{tot}}[\mathrm{bulk}]$ is the total energy for the perfect crystal using an equivalent supercell. The integer $n_{i}$ indicates the number of atoms of type $i$ (host atom or impurity atoms) that have been added to $\left(n_{i}<0\right)$ or removed from $\left(n_{i}<0\right)$ the supercell to form the defect, and the $\mu_{i}$ are the corresponding chemical potentials of these species. For the convenience of calculations, hcp TM metals and $\mathrm{N}_{2}$ molecule in vacuum are taken as the reference states for TM and $\mathrm{N}$ species. This approach has also be used by others to study the defect formation energies [12]. An isolated defect might be attracted to a neighboring defect and form a defect complex. The binding energy $E_{b}$ between two defects $\mathrm{A}$ and $\mathrm{B}$ are obtained by combining the energy of the host TMN, the energies of supercells with single defects A and B, and the energy of the supercell containing the defect complex AB:

$$
E_{b}=E_{f}[\mathrm{~A}]+E_{f}[\mathrm{~B}]-E_{f}[\mathrm{AB}]-E_{\mathrm{tot}}[\mathrm{bulk}] .(2)
$$

Positive (negative) $E_{b}$ 's indicate attraction (repulsion) between defects $\mathrm{A}$ and $\mathrm{B}$. 
The migration energy of $\mathrm{Al}$ and its defect complex in TMNs are calculated by the nudged elastic band method [23] to simulate the minimum energy path that passes through the transition state of a diffusion process.

\section{Results and discussion}

\subsection{Thermodynamic stability of TMNs}

In order to evaluate the thermodynamic stability of $\mathrm{ZrN}$ with respect to $\mathrm{U}-\mathrm{Mo}$ fuel and $\mathrm{Al}$, we calculated the enthalpy of formation for all the possible binary compounds in the UMo-Al-N-Zr system. The enthalpy of formation of binary compounds is calculated by the

formula, $\Delta H_{f}\left(A_{x} B_{y}\right)=\frac{1}{x+y} E_{t o t}\left(A_{x} B_{y}\right)-\frac{x}{x+y} E_{t o t}(A)-\frac{y}{x+y} E_{t o t}(B)$, where $E_{\text {tot }}\left(A_{x} B_{y}\right)$, $E_{\text {tot }}(A)$ and $E_{\text {tot }}(B)$ are the DFT calculated total energies of $A_{x} B_{y}, A$, and $B$, respectively. Our calculations show that most of the compounds exhibit negative formation energies. Only two binary compounds, i.e., $\mathrm{UZr}_{2}$ and $\mathrm{U}_{2} \mathrm{Mo}$, show slightly positive formation energy, indicating that they are thermodynamically unstable with respect to their pure elements. Among all the studied binary compounds, $\mathrm{ZrN}$ show the lowest formation energy, i.e., $-1.75 \mathrm{eV} /$ atom. This is consistent with previous studies showing that transition-metal nitrides are chemically very stable. We also notice that the formation energy of $\mathrm{AlN}$ is close to that of $\mathrm{ZrN}$. We want to point out that the formation energies of all compounds were calculated at zero temperature. Therefore, $\mathrm{Al}$ might react with $\mathrm{ZrN}$ and form $\mathrm{AlN}$ and $\mathrm{Zr}-\mathrm{Al}$ intermetallic compounds at high temperature. Similar for TiN and HfN, DFT calculations show that they are the most stable binary compounds in the U-Mo-Al-N-TM (TM=Ti or Hf) systems. The calculated formation energies are very close to that of $\mathrm{ZrN}$, i.e., $-1.74 \mathrm{eV} /$ atom and $-1.76 \mathrm{eV} /$ atom for TiN and $\mathrm{HfN}$, respectively, as shown in Table 1. Although the DFT predicted formation enthalpies of $\mathrm{ZrN}$ and HfN are slightly underestimated compared to experimental measurements [24-27], the stabilities of the transition-metal nitrides are well captured by the current calculations. 
Therefore, these three transition-metal nitrides might share similar stability when used as a diffusion barrier coating for U-Mo/Al dispersion fuel.

The phase equilibria in the ternary systems of TM-Al-N (TM=Ti, Zr, or Hf) have been investigated by Schuster et al. two decades ago [28-30]. The isothermal sections at high temperatures indicate that ternary compounds, such as $\mathrm{TM}_{3} \mathrm{AlN}$ and $\mathrm{TM}_{5} \mathrm{Al}_{3} \mathrm{~N}$, can form at certain composition ranges. The experiment by Krusin-Elbaum et al. [31] showed that $\mathrm{ZrAl}_{3}$ forms at the interface between $\mathrm{ZrN}$ and $\mathrm{Al}$ at $550{ }^{\circ} \mathrm{C}$. Meanwhile $\mathrm{TiN}$ was observed to be decomposed by $\mathrm{Al}$ at high temperatures [9]. However, it is not clear if they are stable against $\mathrm{Al}$ at low temperatures, such as $150 \sim 200{ }^{\circ} \mathrm{C}$, a typical operating temperature range for research reactors. Based on the ternary phase diagrams of TM-Al-N by Schuster et al. [28-30] and the observed intermetallic compounds $[9,31]$, we proposed the following two potential reactions between $\mathrm{TMN}$ and $\mathrm{Al}$,

$$
\begin{aligned}
& \mathrm{TMN}+\mathrm{Al} \rightarrow \frac{2}{3} \mathrm{AlN}+\frac{1}{3} \mathrm{TM}_{3} \mathrm{AlN}, \\
& \mathrm{TMN}+4 \mathrm{Al} \rightarrow \mathrm{AlN}+\mathrm{TMAl}_{3} .
\end{aligned}
$$

The heats of the chemical reactions were calculated by $\frac{\frac{1}{3} E_{t o t}\left(T M_{3} A l N\right)-\frac{2}{3} E_{t o t}(A l N)-E_{t o t}(T M N)-E_{t o t}(A l)}{3}$ and $\frac{E_{t o t}\left(T M A l_{3}\right)-E_{t o t}(A l N)-E_{t o t}(T M N)-4 E_{t o t}(A l)}{5}$, where $E_{\text {tot }}\left(T M_{3} A l N\right), E_{\text {tot }}\left(T M A l_{3}\right), E_{\text {tot }}(A l N), E_{\text {tot }}(T M N)$, and $E_{\text {tot }}(A l)$ are the total energy of $T M_{3} A l N, T M A l_{3}, A l N, T M N$ and $A l$ calculated by DFT, respectively. As shown in Table 2, the following conclusions can be made from the predicted heats of reaction: (i) The first reaction is thermodynamically less favorable than the second reaction for all the three systems; (ii) $\mathrm{TiN}$ and $\mathrm{ZrN}$ are thermodynamically unstable against $\mathrm{Al}$ due to their exothermic reactions with $\mathrm{Al}$; (iii) $\mathrm{ZrN}$ is the most reactive with $\mathrm{Al}$ among the three; and (iv) $\mathrm{HfN}$ is a thermodynamically stable barrier against $\mathrm{Al}$ due to the endothermic reactions. Consistent with previous study, Lee et al. [9] found that TiN diffusion barrier fails at temperature above $550{ }^{\circ} \mathrm{C}$ due to the reaction with $\mathrm{Al}$. Based on the formed compounds $\mathrm{AlN}$ and $\mathrm{TiAl}_{3}$, they 
proposed the decomposition reaction of $\mathrm{TiN}$ as the second reaction shown above. As to $\mathrm{ZrN}$, Krusin-Elbaum et al.'s study [31] shows that an intermetallic compound $\mathrm{ZrAl}_{3}$ formed due to the reaction between $\mathrm{Al}$ and $\mathrm{ZrN}$ at $550{ }^{\circ} \mathrm{C}$. It was reported that high temperature is required for the reaction between $\mathrm{Al}$ and $\mathrm{ZrN}$ [31]. We note that the rate of this decomposition reaction is controlled by the diffusion of $\mathrm{Al}$ in $\mathrm{ZrN}$ and the newly formed $\mathrm{AlN}$ layers. Although the diffusion of $\mathrm{Al}$ in $\mathrm{ZrN}$ and $\mathrm{AlN}$ is negligible at temperatures below $200{ }^{\circ} \mathrm{C}$, the radiationinduced defects and local temperature gradient can significantly enhance the diffusion of Al. From thermodynamic point of view, $\mathrm{HfN}$ is the most stable diffusion barrier against $\mathrm{Al}$. However, it should be pointed out that HfN may not be a good candidate for nuclear fuel applications due to its higher neutron adsorption cross-section compared to TiN and $\mathrm{ZrN}$.

A potential solution to prevent the decomposition of $\mathrm{TiN}$ and $\mathrm{ZrN}$ by $\mathrm{Al}$ is to add additional layer of immiscible materials between TMN and Al. Since AlN can coexist with both $\mathrm{Al}$ and $\mathrm{ZrN}$, it could be deposited as a second layer on top of the TMN coating to prevent its contact with Al. It has been suggested that layered interface promote the recombination of opposite type of point defects and therefore reduce the accumulative defect density, swelling, and lattice distortion [32]. The chemical stability of interface is an important issue when considering the radiation tolerant multilayer design. To investigate the possible formation of solid solution phase $T M_{1-x} A l_{x} N$, we calculated the enthalpy of solution between TMN and AlN by $\Delta H_{f}\left(T M_{1-x} A l_{x} N\right)=E_{\text {tot }}\left(T M_{1-x} A l_{x} N\right)-(1-x) E_{\text {tot }}(T M N)-x E_{\text {tot }}(A l N)$, where $E_{\text {tot }}\left(T M_{1-x} A l_{x} N\right), E_{\text {tot }}(T M N)$ and $E_{\text {tot }}(A l N)$ are the total energies of $T M_{1-x} A l_{x} N, T M N$ and $A l N$ calculated by DFT, respectively. The predicted positive solution energy indicates that no stable solution phase will form at the interface between TMN and AlN layers. Therefore, TMN and AlN multilayer satisfies the thermodynamic requirement as diffusion barriers. 


\subsection{Kinetic stability of TMNs}

Recent in-pile experiment by Leenaers et al. [7] shows that three types of ILs formed in the $\mathrm{ZrN}$ coated U-Mo/Al dispersion fuel: (i) double coating layer; (ii) "volcano" IL; and (iii) IL covered with coating. The first type of IL could be due to the reaction between $\mathrm{Al}$ and $\mathrm{ZrN}$. High-resolution TEM characterization on the newly formed layers is necessary to confirm their compositions. The second type of IL forms at the surface of U-Mo particle without the pretection of $\mathrm{ZrN}$ coating. It is the classical $(\mathrm{U}, \mathrm{Mo}) \mathrm{Al}_{\mathrm{x}} \mathrm{IL}$ formed at direct contact between $\mathrm{U}$ Mo and Al. The formation of the third type IL is due to radiation-enhanced diffusion of $\mathrm{Al}$ through $\mathrm{ZrN}$ and forms $(\mathrm{U}, \mathrm{Mo}) \mathrm{Al}_{\mathrm{x}}$ below $\mathrm{ZrN}$ coating layer. To understand the formation of the third type of IL, it is important to provide an atomistic scale study of the diffusion mechanism for $\mathrm{Al}$ atoms in $\mathrm{ZrN}$ coating and other proposed diffusion barrier materials.

The most common crystalline form of TMNs is the rock salt structure. In defect-free state, the most stable configuration of $\mathrm{Al}$ atom occupies the tetrahedral interstitial site, i.e., the center of a cubic TMN cell (see Fig. 1 (a) for details). The introduction of an Al atom significantly expands the surrounding cubic lattice by $24.5 \%, 21.8 \%$ and $22.0 \%$, respectively for TiN, ZrN and HfN. The highest lattice distortion of TiN after adding Al can be explained by its smallest lattice constant, compared to $\mathrm{ZrN}$ and $\mathrm{HfN}$. The formation energy of an $\mathrm{Al}$ interstitial is predicted to be $3.81 \mathrm{eV}, 4.25 \mathrm{eV}$, and $5.88 \mathrm{eV}$, respectively for $\mathrm{TiN}, \mathrm{ZrN}$ and HfN. According to ref. [33], the interstitial formation can be roughly divided into two parts: one is from the strain effect, and the other comes from the extra chemical bonding formed between $\mathrm{Al}$ and the surrounding atoms. Using the approach proposed by Shi et al [33], the strain energy can be estimated by comparing the energy difference between the strained TMN and the unstrained TMN structures. The strain energy due to inclusion of an interstitial Al atom is predicted to be 4.9, 5.1 and $5.3 \mathrm{eV}$ for $\mathrm{TiN}, \mathrm{ZrN}$, and $\mathrm{HfN}$, respectively. The highest interstitial formation energy of $\mathrm{Al}$ in $\mathrm{HfN}$ can be explained by the high strain energy and weak bonding between $\mathrm{Al}$ and $\mathrm{N}$. The migration of interstitial $\mathrm{Al}$ atom to a neighboring TMN cell 
proceeds via a transition state, in which $\mathrm{Al}$ atom lies in a (001) plane as shown in Fig. 1 (b).

Figure 1 shows a schematic diagram of the migration of an Al interstitial atom in TMN with migration barrier predicted by the nudged elastic band method [23]. The migration barriers of $\mathrm{Al}$ interstitial atom in $\mathrm{TiN}, \mathrm{ZrN}$ and $\mathrm{HfN}$ are predicted to be $2.42 \mathrm{eV}, 2.19 \mathrm{eV}$ and $1.90 \mathrm{eV}$, respectively, as shown in Table 3 . We note that the trend of the migration barrier of $\mathrm{Al}$ atom in TMN is just opposite that of the formation energy of Al interstitial. This is consistent with the fact that the migration barrier comes from the energy difference between the transition state (Fig. 1(b)) and the equilibrium state (Fig. 1(a) and (c)). The highest migration energy of interstitial $\mathrm{Al}$ in TiN can be explained by the smallest lattice constant of TiN among the three, leading to the highest relative energy of the transition state. From the kinetic point of view, TiN is the most efficient diffusion barrier against $\mathrm{Al}$ among the three. Meanwhile, Tsetseris et al. $[11,14,15]$ have systematically studied the diffusion of $\mathrm{Cu}$ atom in $\mathrm{TiN}, \mathrm{ZrN}$ and $\mathrm{HfN}$. Their predicted migration energy of $\mathrm{Cu}$ interstitial atom follows the same trend as $\mathrm{Al}$ in these three systems, although the migration energy of $\mathrm{Cu}$ in TMN is much lower as shown in Table 3.

In experiment, the rock salt structured TMN phase is found to be stable for a wide range of $\mathrm{N}$ stoichiometry $x$, and the diffusion barrier properties can be optimized by tuning the $x$ values. In the following section, we will discuss the role of non-stoichiometry on the migration of $\mathrm{Al}$ in TMNs. In sub-stoichiometric $\mathrm{TMN}_{1-x}, \mathrm{~N}$ vacancy $\left(\mathrm{N}_{\mathrm{vac}}\right)$ is the most prominent form of single defects; while the formation energy of TM interstitial is considerable higher than other defects (see Table 4 for more details). Due to the relative large atom size of TMs, TM interstitials are energetically more stable to form the TM-TM dumbbell configuration along the $<111>$ direction instead of occupying the tetrahedral interstitial site. In hyper-stoichiometric $\mathrm{TMN}_{1+x}, \mathrm{~N}$ interstitial $\left(\mathrm{N}_{\text {int }}\right)$ and $\mathrm{TM}$ vacancy $\left(\mathrm{TM}_{\mathrm{vac}}\right)$ can coexist due to their similar formation energies, as shown in Table 4. Consistent with previous studies by Tsetseris et al., the most stable form of $\mathrm{N}$ interstitial in TiN form a N-N dumbbell along <110> 
direction, while the $\mathrm{N}$ interstitial atom prefers to occupy the tetrahedral interstitial site in $\mathrm{ZrN}$ and HfN. It should be pointed out that the defect formation energy in TMN is a function of the chemical potentials of $\mathrm{N}$ and TM species. As mentioned in the section 2, hcp TM metal and $\mathrm{N}_{2}$ molecule in vacuum were used as the reference states for TM and $\mathrm{N}$ species in this work. Therefore, the predicted vacancy formation energies of $\mathrm{N}$ and TM shown in Table 4 are the upper bound of the vacancy formation energies in the TMN systems, while the predicted formation energies of $\mathrm{N}$ and TM interstitials are the lower bound. Taking $\mathrm{N}$ defects in $\mathrm{ZrN}$ as an example, the $\mathrm{N}$ vacancy formation energy is higher than that of $\mathrm{N}$ interstitial at the higher bound of $\mu_{\mathrm{N}}$, i.e., the $\mu_{\mathrm{N}, \mathrm{gas}}$-dominated region. With decreasing $\mu_{N}, \mathrm{~N}$ vacancy formation energy decreases while the formation energy of $\mathrm{N}$ interstitial increases. Since the chemical potentials of $\mathrm{N}$ and $\mathrm{Zr}$ are not independent in $\mathrm{ZrN}$ system, the lower bound of $\mu_{\mathrm{N}}$ is limited by $\mu_{\mathrm{N}}=\mu_{\mathrm{ZrN}}-\mu_{\mathrm{Zr} \text {,metal }}$, where $\mu_{\mathrm{ZrN}}$ equals the total energy of a bulk $\mathrm{ZrN}$ per molecule and $\mu_{\mathrm{Zr}}$ represents the total energy of a bulk hcp metal per atom. As shown in Figure 2, the formation energy of an $\mathrm{N}$ intestinal becomes much higher than that of $\mathrm{N}$ vacancy at the lower bound of

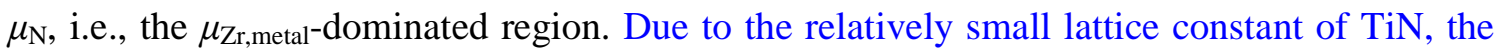
formation energy of $\mathrm{N}$ interstitial in TiN is much higher than that of $\mathrm{N}$ vacancy, while the $\mathrm{N}$ intestinal formation energy in $\mathrm{ZrN}$ and $\mathrm{HfN}$ is slightly lower than that of $\mathrm{N}$ vacancy. The currently predicted defect formation energies of $\mathrm{N}$ in $\mathrm{TiN}, \mathrm{ZrN}$ and $\mathrm{HfN}$ are overall consistent with the previous study by Tsetseris et al. [12] except for $\mathrm{N}$ interstitial in $\mathrm{ZrN}$. In their study, the formation energy of $\mathrm{N}$ interstitial was predicted to be higher than $\mathrm{N}$ vacancy. Such a discrepancy is due to the fact that the lattice constants were fixed during their calculations, which can lead to overestimated defect formation energies, especially for interstitials.

As an $\mathrm{Al}$ interstitial atom hops around in TMN lattice, it may encounter $\mathrm{N}$ or TM vacancy and therefore be trapped at the vacancy site to form a substitutional atom at $\mathrm{N}\left(\mathrm{Al}_{\mathrm{N}}\right)$ or $\mathrm{TM}\left(\mathrm{Al}_{\mathrm{TM}}\right)$ lattice site. As shown in Table 5, the calculated binding energies of $\mathrm{Al}$ interstitial and vacancy defects are all positive, implying that the interaction between two 
defects is attractive and the formation of defect complex is energetically favorable. We also notice that the attraction between $\mathrm{Al}$ interstitial and TM vacancy is much higher than that with $\mathrm{N}$ vacancy. When an $\mathrm{Al}$ interstitial atom is trapped in vacancy sites, it becomes essentially immobile unless either heated at extremely high temperatures, or when there is another vacancy around. In sub-stoichiometric $\mathrm{TMN}_{1-x}$, the trapped $\mathrm{Al}$ atom can attract a neighboring $\mathrm{N}$ vacancy and form a stable defect complex $\mathrm{Al}_{\mathrm{N}}-\mathrm{N}_{\text {vac }}$ in $\mathrm{ZrN}$ and $\mathrm{HfN}$ with positive binding energy as shown in Table 6, while such defect complex is unstable in TiN. The Al atom trapped at $\mathrm{N}$ site shows a weak repulsion with $\mathrm{TM}$ vacancy in $\mathrm{ZrN}$ and $\mathrm{HfN}$ or zero interaction with Ti vacancy in TiN, therefore it won't form stable defect complex with TM vacancy in TMN systems. The migration of $\mathrm{Al}_{\mathrm{N}}-\mathrm{N}_{\mathrm{vac}}$ defect complex requires at least two steps, as shown in Figure 3. In the first step, the trapped $\mathrm{Al}$ atom moves to the neighboring $\mathrm{N}$ vacancy site and leaves behind a new vacancy. In the second step, a nearby $\mathrm{N}$ atom moves to this new formed vacancy site, or equivalently, the vacancy hops to a nearby $\mathrm{N}$ site. Repetition of this two-step sequence allows for the diffusion of $\mathrm{Al}_{\mathrm{N}}-\mathrm{N}_{\text {vac }}$. Taking $\mathrm{ZrN}$ as an example, the migration energy of the $\mathrm{Al}$ atom to a neighboring $\mathrm{N}$ vacancy site in the first step is about 2.35 $\mathrm{eV}$, while the migration energy of the second step is about $3.32 \mathrm{eV}$. Since the energy cost of the second step is higher than the first one, the effective energy barrier for the migration of the defect complex $\mathrm{Al}_{\mathrm{N}}-\mathrm{N}_{\text {vac }}$ in $\mathrm{ZrN}$ is $3.32 \mathrm{eV}$, which is considerable higher than that of an isolated $\mathrm{Al}$ interstitial atom $(2.19 \mathrm{eV})$. Therefore, $\mathrm{N}$ vacancy, i.e., sub-stoichiometry, could be beneficial to slow down the diffusion of $\mathrm{Al}$ atoms in $\mathrm{ZrN}$ and $\mathrm{HfN}$. In hyper-stoichiometric $\mathrm{TMN}_{1+x}$, the $\mathrm{Al}$ interstitial atom can attract a nearby $\mathrm{N}$ interstitial and form a stable defect complex $\mathrm{Al}_{\text {int }}-\mathrm{N}_{\text {int }}$ as shown in Fig. 4(a), which is suggested by the positive binding energies between $\mathrm{Al}$ and $\mathrm{N}$ interstitials shown in Table 5. The highest binding energy of $\mathrm{Al}$ and $\mathrm{N}$ interstitial atoms in TiN indicates that the concentration of such defect complex will be the highest among the three nitride systems. There are several possible pathways for the migration of the defect complex $\mathrm{Al}_{\text {int }}-\mathrm{N}_{\text {int }}$, with one of them shown in Figure 4. Figure 4(b) depicts an 
intermediate structure in the minimum energy diffusion pathway. The energy of the intermediate structure is about $0.87 \mathrm{eV}$ higher than that of the most stable defect complex. The first step shows much higher migration energy than that of the second step, therefore the ratelimiting step in the migration process in shown Fig. 4 is the transformation from (a) to (b). The migration energy of such complex is lower than that of an isolated Al interstitial. We want to point out that non-stoichiometry can increase the number of intrinsic defects, such as $\mathrm{N}$ vacancies and interstitials, leading to increased diffusion of Al impurity. However, slight sub-stoichiometry could be beneficial to slowing down the diffusion Al in TMNs by trapping $\mathrm{Al}$ at $\mathrm{N}$ or TM lattice site or forming stable defect complexes. It is worth noting that we only studied the diffusion of $\mathrm{Al}$ atom through lattice, which is the dominant diffusion mechanism at high temperatures. However, atom diffusion through grain boundary or surface is more important at low temperatures, which will be the goal of our future study.

\section{Summary}

We investigated the thermodynamic and kinetic stability of three transitional-metal nitride systems as diffusion barriers against Al for U-Mo dispersion fuel applications. Although $\mathrm{ZrN}$ and TiN are the most stable binary phase in the U-Mo-Al-TM-N system, they can be decomposed by $\mathrm{Al}$ at high temperature due to chemical reactions. The rate of such reaction is determined by the diffusion rate of $\mathrm{Al}$ through $\mathrm{TMN}$ and the newly formed $\mathrm{AlN}$ layers. A multilayer coating scheme, such as TMN/AlN, was proposed to avoid the decomposition reaction of TMN diffusion barriers. As a comparison, $\mathrm{HfN}$ is predicted to be a thermodynamically stable diffusion barrier material for Al. However, due to its high neutron absorption cross-section, HfN is not a good choice for nuclear fuel applications. To evaluate the kinetic stability of TMNs, we also provided an atomistic study of the diffusion mechanism of $\mathrm{Al}$ in $\mathrm{TiN}, \mathrm{ZrN}$ and $\mathrm{HfN}$. In defect-free TMN samples, the $\mathrm{Al}$ atom prefers to occupy the tetrahedral interstitial site in the rock salt structured TMN. The migration of Al interstitial 
atom in TiN was found to be most difficult. In sub-stoichiometric TMN samples, Al interstitial atom can be easily trapped by a neighboring $\mathrm{N}$ or TM vacancy and becomes an idle atom unless additional $\mathrm{N}$ vacancy appears at its nearby site. In hyper-stoichiometric TMN samples, $\mathrm{Al}$ interstitial atom can attract a nearby $\mathrm{N}$ interstitial atom and form stable defect

complex $\mathrm{Al}_{\text {int }}-\mathrm{N}_{\text {int }}$. The migration barrier of the defect complex $A l_{N}-\mathrm{N}_{\text {vac }}$ is higher than that of an isolated Al interstitial atom. Therefore, slight sub-stoichiometry can be beneficial to slowing down the diffusion of $\mathrm{Al}$ atoms in TMNs, especially for $\mathrm{ZrN}$ and $\mathrm{HfN}$.

\section{Acknowledgement}

This work is sponsored by the U.S. Department of Energy, National Nuclear Safety Administration (NNSA), Office of Material Management and Minimization (NA-23) Reactor Conversion Program. Use of the Center for Nanoscale Materials, an Office of Science user facility, was supported by the U. S. Department of Energy, Office of Science, Office of Basic Energy Sciences, under Contract No. DE-AC02-06CH11357. We gratefully acknowledge the computing resources provided on Blues, a high-performance computing cluster operated by the Laboratory Computing Resource Center at Argonne National Laboratory. 


\section{References}

[1] M. K. Meyer et al., Journal of Nuclear Materials 304, 221 (2002).

[2] Y. S. Kim, in Comprehensive Nuclear Materials, edited by R. J. M. Konings (Elsevier, Oxford, 2012), pp. 391.

[3] Y. S. K. G.L. Hofman, M.R. Finlay, J.L. Snelgrove, S.L. Hayes, M.K. Meyer, C.R. Clark, F. Huet, , in the 25th International Meeting on Reduced Enrichment for Research and Test Reactors (RERTR)Chicago, Illinois, 2003).

[4] G. L. H. Y.S. Kim, H.J. Ryu, J. Rest, in the 2005 RERTR International MeetingBoston, USA, 2005).

[5] A. Leenaers, S. Van den Berghe, and C. Detavernier, Journal of Nuclear Materials 440, 220 (2013).

[6] S. Van den Berghe, W. Van Renterghem, and A. Leenaers, Journal of Nuclear Materials 375, 340 (2008).

[7] A. Leenaers, Ph.D. Thesis, University of Ghent-SCK•CEN, 2014.

[8] M. A. Nicolet, and M. Bartur, Journal of Vacuum Science \& Technology 19, 786 (1981).

[9] H.-J. Lee et al., Journal of Applied Physics 86, 3096 (1999).

[10] C. Lee, and Y.-L. Kuo, JOM 59, 44 (2007).

[11] L. Tsetseris et al., Physical Review Letters 99, 125503 (2007).

[12] L. Tsetseris et al., Physical Review B 76, 224107 (2007).

[13] L. Tsetseris et al., Physical Review B 78, 094111 (2008).

[14] L. Tsetseris, S. Logothetidis, and S. T. Pantelides, Applied Physics Letters 94, 161903 (2009).

[15] L. Tsetseris, S. Logothetidis, and S. T. Pantelides, Surface and Coatings Technology 204, 2089 (2010).

[16] M. Pukari, P. Olsson, and N. Sandberg, Journal of Nuclear Materials 438, 7 (2013).

[17] G. Kresse, and J. Furthmüller, Phys. Rev. B 54, 11169 (1996).

[18] G. Kresse, and D. Joubert, Phys. Rev. B 59, 1758 (1999).

[19] J. P. Perdew, K. Burke, and M. Ernzerhof, Phys. Rev. Lett. 77, 3865 (1996).

[20] H. Höchst et al., Physical Review B 25, 7183 (1982).

[21] A. N. Christensen, Acta Chemica Scandinavica A 29, 563 (1975).

[22] H.-S. Seo et al., Journal of Applied Physics 96, 878 (2004).

[23] G. Henkelman, B. P. Uberuaga, and H. Jónsson, The Journal of Chemical Physics 113, 9901 (2000).

[24] G. L. Humphrey, Journal of the American Chemical Society 73, 2261 (1951).

[25] G. L. Humphrey, Journal of the American Chemical Society 75, 2806 (1953).

[26] A. D. Mah, and N. L. Gellert, Journal of the American Chemical Society 78, 3261 (1956).

[27] V. I. Ivashchenko, P. E. A. Turchi, and V. I. Shevchenko, Condensed Matter Physics 16, 1 (2013).

[28] J. C. Schuster, and J. Bauer, Journal of Solid State Chemistry 53, 260 (1984).

[29] J. C. Schuster, J. Bauer, and J. Debuigne, Journal of Nuclear Materials 116, 131 (1983).

[30] J. C. Schuster, and J. Bauer, Journal of Nuclear Materials 120, 133 (1984).

[31] L. Krusin-Elbaum et al., Thin Solid Films 104, 81 (1983).

[32] M. Milosavljević et al., Thin Solid Films 544, 562 (2013).

[33] X. Shi et al., Physical Review B 75, 235208 (2007). 


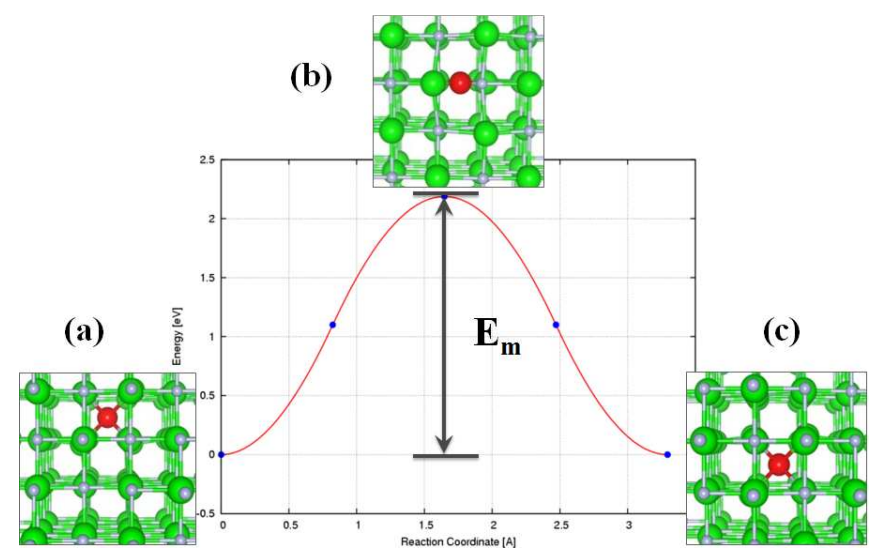

Figure 1. Migration of Al interstitial atom in TMN. Large green balls represent $\mathrm{Zr}$ atoms, small grey balls represent $\mathrm{N}$ atoms and red ball represents $\mathrm{Al}$ atom. 


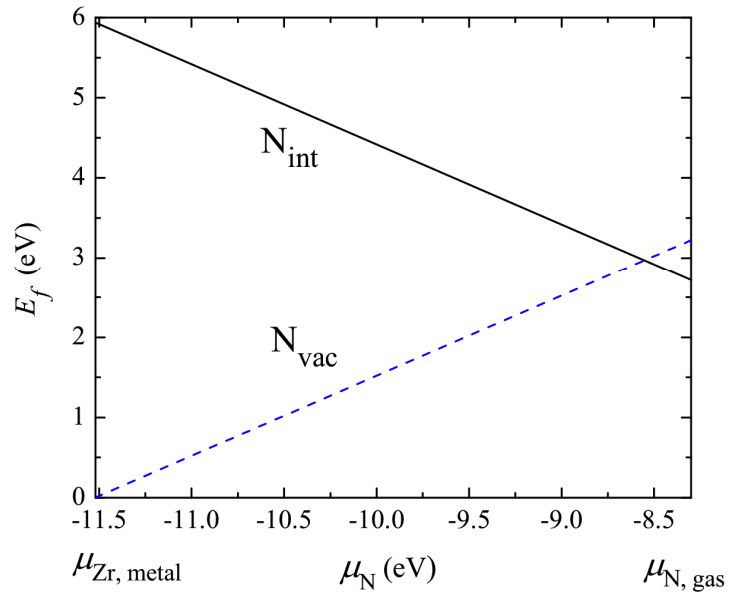

Figure 2. The predicted defect formation energies of $\mathrm{N}$ vacancy and interstitial in $\mathrm{ZrN}$ as a function of $\mathrm{N}$ chemical potential. The lower bound of $\mathrm{N}$ chemical potential is limited by $\mu_{\mathrm{N}}$ $=\mu_{\mathrm{ZrN}}-\mu_{\mathrm{Zr} \text {,metal }}$, while the higher bound of $\mathrm{N}$ chemical potential comes from that of $\mathrm{N}_{2}$ molecule in vaccum. 


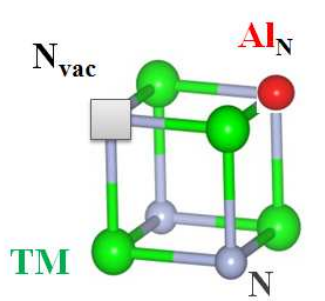

(a)

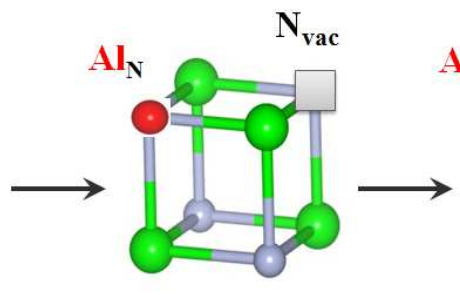

(b)

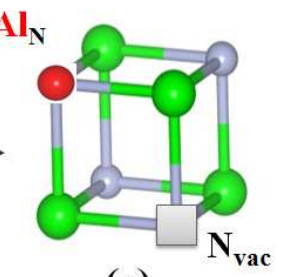

(c)

Figure 3. Schematic of the migration of a defect complex $A l_{N}-N_{v a c}$ in sub-stoichiometric $\mathrm{TMN}_{1-x}$. Large green balls represent TM atoms, small grey balls represent $\mathrm{N}$ atoms and red ball represents $\mathrm{Al}$ atom. 


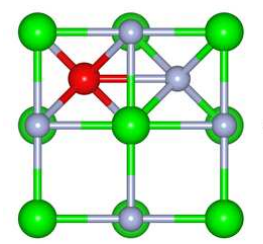

(a)

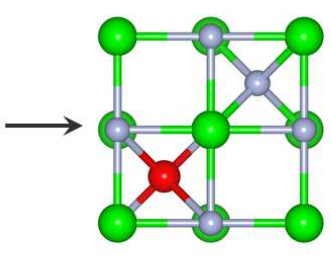

(b)

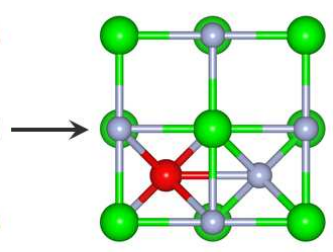

(c)

Figure 4. Schematic of the migration of a defect complex $\mathrm{Al}_{\text {int }}-\mathrm{N}_{\text {int }}$ in hyperstoichiometric $\mathrm{TMN}_{1+x}$. Large green balls represent $\mathrm{TM}$ atoms, small grey balls represent $\mathrm{N}$ atoms and red ball represents $\mathrm{Al}$ atom. 
Table 1. Enthalpy of formation $\Delta H_{\mathrm{f}}(\mathrm{eV} /$ atom $)$ of TiN, $\mathrm{ZrN}$ and $\mathrm{HfN}$

\begin{tabular}{lcll}
\hline \hline Phase & $\Delta H_{\mathrm{f}}$ & Methodology & Reference \\
\hline TiN & -1.74 & VASP-GGA & This work \\
& -1.74 & Quantum ESPPRESSO-GGA & Ivashchenko [27] \\
& -1.74 & Experiment & Humphrey [24] \\
ZrN & -1.75 & VASP-GGA & This work \\
& -1.76 & Quantum ESPPRESSO-GGA & Ivashchenko [27] \\
& -1.89 & Experiment & Mah [26] \\
HfN & -1.76 & VASP-GGA & This work \\
& -1.91 & Experiment & Humphrey [25] \\
\hline \hline
\end{tabular}

Table 2. Heats of reaction $\Delta_{R} H(\mathrm{~kJ} / \mathrm{mol}$-atom) between TMN and Al by DFT.

\begin{tabular}{lccc}
\hline \hline Chemical reactions & TiN & ZrN & HfN \\
\hline TMN $+\mathrm{Al} \rightarrow \frac{2}{3} \mathrm{AlN}+\frac{1}{3} \mathrm{TM}_{3} \mathrm{AlN}$ & 0.24 & -0.75 & 66.37 \\
$\mathrm{TMN}+4 \mathrm{Al} \rightarrow \mathrm{AlN}+\mathrm{TMAl}_{3}$ & -14.95 & -20.04 & 18.82 \\
\hline \hline
\end{tabular}

Table 3. Formation energy $E_{f}$ and migration barrier $E_{m}$ of Al interstitial in TMN by DFT.

\begin{tabular}{cccc}
\hline \hline TMN & $E_{\mathrm{f}}(\mathrm{eV})$ & $E_{m}-\mathrm{Al}(\mathrm{eV})$ & $E_{m}-\mathrm{Cu}(\mathrm{eV})^{\mathrm{a}}$ \\
\hline TiN & 3.81 & 2.42 & 1.4 \\
$\mathrm{ZrN}$ & 4.25 & 2.19 & 1.0 \\
$\mathrm{HfN}$ & 5.88 & 1.90 & 0.9 \\
\hline
\end{tabular}

${ }^{\mathrm{a}}$ References $[11, \overline{\overline{14,15}}$ 
Table 4. Formation energy of point defects in TMN by DFT.

\begin{tabular}{ccccc}
\hline \hline TMN & $\mathrm{N}_{\text {vac }}(\mathrm{eV})$ & $\mathrm{N}_{\text {int }}(\mathrm{eV})$ & $\mathrm{TM}_{\text {vac }}(\mathrm{eV})$ & $\mathrm{TM}_{\text {int }}(\mathrm{eV})$ \\
\hline $\mathrm{TiN}$ & 2.53 & 4.74 & 3.11 & 7.31 \\
$\mathrm{ZrN}$ & 3.22 & 2.72 & 2.91 & 10.19 \\
$\mathrm{HfN}$ & 3.34 & 2.62 & 1.83 & 12.42 \\
\hline \hline
\end{tabular}

Table 5. Binding energy between $\mathrm{Al}_{\text {int }}$ and intrinsic defects in TMN by DFT.

\begin{tabular}{cccc}
\hline \hline TMN & $\mathrm{Al}_{\text {int }}-\mathrm{N}_{\text {vac }}(\mathrm{eV})$ & $\mathrm{Al}_{\text {int }}-\mathrm{TM}_{\text {vac }}(\mathrm{eV})$ & $\mathrm{Al}_{\text {int }}-\mathrm{N}_{\text {int }}(\mathrm{eV})$ \\
\hline $\mathrm{TiN}$ & 1.50 & 8.78 & 1.99 \\
$\mathrm{ZrN}$ & 3.37 & 8.24 & 1.32 \\
$\mathrm{HfN}$ & 4.43 & 9.00 & 1.75 \\
\hline \hline
\end{tabular}

Table 6. Binding energy between trapped $\mathrm{Al}_{\mathrm{N}}$ and vacancy defects in TMN by DFT.

\begin{tabular}{ccc}
\hline \hline TMN & $\mathrm{Al}_{\mathrm{N}}-\mathrm{N}_{\text {vac }}(\mathrm{eV})$ & $\mathrm{Al}_{\mathrm{N}}-\mathrm{TM}_{\mathrm{vac}}(\mathrm{eV})$ \\
$\mathrm{TiN}$ & -0.16 & 0.00 \\
$\mathrm{ZrN}$ & 0.24 & -0.10 \\
$\mathrm{HfN}$ & 0.26 & -0.10 \\
\hline
\end{tabular}

